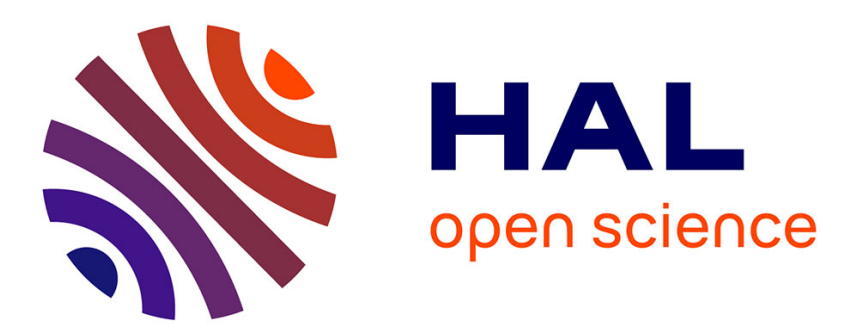

\title{
A locking-free discontinuous Galerkin method for linear elasticity in locally nearly incompressible heterogeneous media
}

Daniele Antonio Di Pietro, Serge Nicaise

\section{- To cite this version:}

Daniele Antonio Di Pietro, Serge Nicaise. A locking-free discontinuous Galerkin method for linear elasticity in locally nearly incompressible heterogeneous media. Applied Numerical Mathematics, 2012, 63, pp.105-116. 10.1016/j.apnum.2012.09.009 • hal-00685020

\section{HAL Id: hal-00685020 \\ https://hal.science/hal-00685020}

Submitted on 3 Apr 2012

HAL is a multi-disciplinary open access archive for the deposit and dissemination of scientific research documents, whether they are published or not. The documents may come from teaching and research institutions in France or abroad, or from public or private research centers.
L'archive ouverte pluridisciplinaire HAL, est destinée au dépôt et à la diffusion de documents scientifiques de niveau recherche, publiés ou non, émanant des établissements d'enseignement et de recherche français ou étrangers, des laboratoires publics ou privés. 


\title{
A locking-free discontinuous Galerkin method for linear elasticity in locally nearly incompressible heterogeneous media
}

\author{
Daniele A. Di Pietro ${ }^{\mathrm{a}}$, Serge Nicaise ${ }^{\mathrm{b}}$ \\ ${ }^{a}$ Department of Applied Mathematics, IFP Energies nouvelles, 1 \& 4 avenue Bois Préau, 92852 \\ Rueil-Malmaison, France \\ ${ }^{b}$ LAMAV, Université de Valenciennes et du Hainaut Cambrésis, Le Mont Houy, 59313 Valenciennes CEDEX 9, \\ France
}

\begin{abstract}
In this work we consider the problem of numerical locking in composite materials featuring quasiincompressible and compressible sections. More specifically, we start by extending a classical regularity estimate for the $H^{1}$-norm of the divergence of the displacement field to the heterogeneous case. The proof is based on a reformulation of the elasticity problem as a Stokes system with nonzero divergence constraint. This result is then used to design a locking-free discontinuous Galerkin method. The key point is to make sure that the multiplicative constant in the estimate of the convergence rate uniquely depend on the this bounded quantity. Thanks to a fine tuning of the penalty term, the lower bound for the penalty parameter appearing in the method is simply expressed in terms of the space dimension. To conclude, numerical validation of the theoretical results is provided.
\end{abstract}

Keywords: Linear elasticity, composite materials, locking-free method, discontinuous Galerkin method

\section{Introduction}

This work addresses the problem of numerical locking in heterogeneous, locally nearly incompressible media by revisiting the classical works of Brenner and Sung [1] and Hansbo and Larson [2, 3]; see also Wihler [4]. More specifically, let $\Omega \subset \mathbb{R}^{d}, d \geq 2$, be a bounded polygonal domain. We do not assume that $\Omega$ is a Lipschitz domain to include the presence of cracks in our analysis. We consider the linear elasticity problem

$$
\begin{aligned}
-\operatorname{div} \underline{\underline{\sigma}}(\mathbf{u})=\mathbf{f} & \text { in } \Omega, \\
\mathbf{u}=\mathbf{0} & \text { on } \partial \Omega,
\end{aligned}
$$

where $\mathbf{f} \in L^{2}(\Omega)^{d}$, $\mathbf{u}$ denotes the vector-valued displacement field, and, for all $\mathbf{v} \in H^{1}(\Omega)^{d}$,

$$
\underline{\underline{\sigma}}(\mathbf{v}):=2 \mu \underline{\underline{\epsilon}}(\mathbf{v})+\lambda \operatorname{div} \mathbf{v} \underline{\underline{I_{d}}}, \quad \underline{\underline{\epsilon}}(\mathbf{v}):=\frac{1}{2}\left(\nabla \mathbf{v}+\nabla \mathbf{v}^{t}\right) .
$$

Here, $\mu$ and $\lambda$ are scalar-valued fields corresponding to Lamé's parameters such that

$$
0<\underline{\mu} \leq \mu \leq \bar{\mu}<+\infty, \quad 0<\underline{\lambda} \leq \lambda \leq \bar{\lambda} .
$$

We focus on heterogeneous media for which there exists a partition $P_{\Omega}=\left\{\Omega_{i}\right\}_{1 \leq i \leq N_{\Omega}}$ of $\Omega$ into polyhedral subdomains such that $\mu$ and $\lambda$ are piecewise constant on $P_{\Omega}$. Our goal is to design a locking-free discontinuous Galerkin method, meaning that the error estimate should not blow up when $\underline{\lambda}$ is fixed and large enough and $\bar{\lambda} \rightarrow+\infty$. The robustness with respect to $\mu$ is not considered herein, since large heterogeneity ratios $\bar{\mu} / \underline{\mu}$ do not correspond to physically relevant situations. 
Let $H^{l}\left(P_{\Omega}\right):=\left\{v \in L^{2}(\Omega) \mid v_{\mid \Omega_{i}} \in H^{l}\left(\Omega_{i}\right), 1 \leq i \leq N_{\Omega}\right\}$. An instrumental result to prove a locking-free error estimate is to show that the quantity

$$
\mathcal{N}_{\mathbf{u}}:=\left(\|\mathbf{u}\|_{H^{2}\left(P_{\Omega}\right)^{d}}^{2}+|\lambda \operatorname{div} \mathbf{u}|_{H^{1}\left(P_{\Omega}\right)}^{2}\right)^{1 / 2}
$$

stays bounded when $\bar{\lambda} \rightarrow+\infty$. This is proved in of Sect. 2 in the two-dimensional case $d=2$. While this result is a generalization of [1, eq. (2.18)] to the heterogeneous case, the techniques used in the proof are new and are inspired by the recent work of Nicaise and Mercier [5. The key idea is to reformulate the elasticity problem in terms of a transmission Stokes problem by introducing a fictitious pressure defined as the product of the first Lamé parameter by the divergence of the displacement field. The bound for (2) then follows from an energy estimate for the elasticity problem together with the local regularity of the solutions to the Stokes problem.

With an estimate for $\mathcal{N}_{\mathbf{u}}$ at hand, the goal of Sect. 3 is to design a discontinuous Galerkin $(\mathrm{dG})$ method based on piecewise affine functions and satisfying an estimate of the form

$$
\left\|\mathbf{u}-\mathbf{u}_{h}\right\|_{\mu, \lambda} \leq C_{\mu} \mathcal{N}_{\mathbf{u}} h,
$$

where $\|\cdot\|_{\mu, \lambda}$ is the energy-like norm defined in (14), $\mathbf{u}_{h}$ is the discrete solution, and $C_{\mu}$ denotes a constant depending on $\mu$ and on $\|\mathbf{f}\|_{L^{2}(\Omega)^{d}}$ but not on $\lambda$. A key point in this direction is to make sure that $\lambda$ only appears either in terms involving a product by the divergence of $\mathbf{u}_{h}$, or in terms that can be cancelled by an appropriate choice of the interpolator in the error estimate. This is achieved here by designing the penalty term so that (i) the weak coercivity (nonnegativity) of the discrete bilinear form is obtained by penalizing piecewise constant jump liftings in a leastsquare fashion with a user-dependent parameter $\eta$; (ii) full coercivity is achieved by a standard interior penalty term with a coefficient that solely depends on $\mu$ and on the mesh size $h$. This term is required since penalizing the average component of the jumps is insufficient to use the discrete Korn inequality derived by Brenner [6]. Unlike [3], the lower bound for the parameter $\eta$ is simply expressed in terms of the space dimension, i.e., no undetermined trace constant appears. The idea of penalizing using jump liftings can be traced back to the seminal works of Bassi and Rebay [7, 8]. An explicit formula is provided to ease the practical implementation of liftings, and the flux formulation of the method is briefly discussed for the sake of completeness in Sect. 3.5. Moreover, the convergence of the method to minimal regularity solutions is addressed in Sect. 3.6.

The numerical assessment of the proposed method is performed in Sect. 4 using (i) the closed cavity problem of [3] and (ii) the exact solution derived by Hongjun, Zhifei, and Taotao [9] for a composite multi-layer infinite cylinder. The results confirm that no loss of accuracy is observed in the incompressible limit.

\section{A regularity result}

Letting $\mathbf{U}:=H_{0}^{1}(\Omega)^{d}$, the weak formulation of problem (1) reads: Find $\mathbf{u} \in \mathbf{U}$ such that

$$
a(\mathbf{u}, \mathbf{v})=\int_{\Omega} \mathbf{f} \cdot \mathbf{v} \quad \forall \mathbf{v} \in \mathbf{U}
$$

where $a(\mathbf{u}, \mathbf{v}):=\int_{\Omega} \underline{\underline{\sigma}}(\mathbf{u}): \underline{\underline{\epsilon}}(\mathbf{v})$ and, for two second-order tensors $\underline{\underline{\alpha}}$ and $\underline{\underline{\beta}}$, we have denoted $\underline{\underline{\alpha}}: \underline{\underline{\beta}}:=$ $\sum_{1 \leq i, j \leq d} \alpha_{i j} \beta_{i j}$. In what follows we prove an a priori estimate for the quantity $\mathcal{N}_{\mathbf{u}}$ defined by (2) in the two-dimensional case $d=2$.

Lemma 1 (Stability estimate). If $\mathbf{u} \in \mathbf{U}$ is the unique solution of $(3)$ with $\mathbf{f} \in L^{2}(\Omega)^{d}$, then the following energy estimate holds

$$
\left\|(2 \mu)^{1 / 2} \underline{\underline{\epsilon}}(\mathbf{u})\right\|_{L^{2}(\Omega)^{d, d}}+\left\|\lambda^{1 / 2} \operatorname{div} \mathbf{u}\right\|_{L^{2}(\Omega)} \leq \frac{C_{\Omega}}{\underline{\mu}^{1 / 2}}\|\mathbf{f}\|_{L^{2}(\Omega)^{d}},
$$

where $C_{\Omega}$ is the product of the Poincaré and Korn constants. 
Proof. Taking $\mathbf{u}$ as a test function in (3) it is inferred $\left\|(2 \mu)^{1 / 2} \underline{\underline{\epsilon}}(\mathbf{u})\right\|_{L^{2}(\Omega) d, d}^{2}+\left\|\lambda^{1 / 2} \operatorname{div} \mathbf{u}\right\|_{L^{2}(\Omega)}^{2}=$ $a(\mathbf{u}, \mathbf{u})=\int_{\Omega} \mathbf{f} \cdot \mathbf{u}$. Using the Cauchy-Schwarz, Poincaré, Korn, and Young's inequalities it is inferred

$$
\int_{\Omega} \mathbf{f} \cdot \mathbf{u} \leq\|\mathbf{f}\|_{L^{2}(\Omega)^{d}}\|\mathbf{u}\|_{L^{2}(\Omega)^{d}} \leq C_{\Omega}\|\mathbf{f}\|_{L^{2}(\Omega)^{d}}\|\underline{\underline{\epsilon}}(\mathbf{u})\|_{L^{2}(\Omega)^{d, d}} \leq \frac{1}{2} \frac{C_{\Omega}^{2}}{2 \underline{\mu}}\|\mathbf{f}\|_{L^{2}(\Omega)^{d}}^{2}+\frac{1}{2}\left\|(2 \mu)^{1 / 2} \underline{\underline{\epsilon}}(\mathbf{u})\right\|_{L^{2}(\Omega)^{d, d}}^{2} .
$$

The conclusion follows.

To proceed, we derive an alternative weak formulation which relates the solution of $(3)$ to that of a transmission Stokes problem. To this purpose, let $L_{0}^{2}(\Omega):=\left\{p \in L^{2}(\Omega):\langle p\rangle_{\Omega}=0\right\}$, where, for a function $\phi$ integrable on $\Omega,\langle\phi\rangle_{\Omega}:=\frac{1}{|\Omega|_{d}} \int_{\Omega} \phi$. We introduce the bilinear forms $a_{0} \in \mathcal{L}(\mathbf{U} \times \mathbf{U}, \mathbb{R})$ and $b_{0} \in \mathcal{L}\left(\mathbf{U} \times L_{0}^{2}(\Omega), \mathbb{R}\right)$ defined as follows:

$$
a_{0}(\mathbf{w}, \mathbf{v}):=\int_{\Omega} 2 \mu \underline{\underline{\epsilon}}(\mathbf{w}): \underline{\underline{\epsilon}}(\mathbf{v}), \quad b_{0}(\mathbf{w}, q):=-\int_{\Omega} \operatorname{div} \mathbf{w} q .
$$

Then, letting

$$
p:=-\lambda \operatorname{div} \mathbf{u} \in L^{2}(\Omega), \quad \tilde{p}:=p+\langle\lambda \operatorname{div} \mathbf{u}\rangle_{\Omega} \in L_{0}^{2}(\Omega),
$$

we infer that $(\mathbf{u}, \tilde{p}) \in \mathbf{U} \times L_{0}^{2}(\Omega)$ is the unique solution of the transmission Stokes problem

$$
\begin{array}{rlrl}
a_{0}(\mathbf{u}, \mathbf{v})+b_{0}(\mathbf{v}, \tilde{p}) & =\int_{\Omega} \mathbf{f} \cdot \mathbf{v} & & \forall \mathbf{v} \in \mathbf{U}, \\
b_{0}(\mathbf{u}, q) & =\int_{\Omega} g q & \forall q \in L_{0}^{2}(\Omega),
\end{array}
$$

with $g=-\operatorname{div} \mathbf{u}$. We assume that $\mathbf{u} \in H^{2}\left(P_{\Omega}\right)^{d}$, and that problem (6) satisfies the optimal regularity shift, meaning that the regularity $(\mathbf{f}, g) \in L^{2}(\Omega)^{d} \times H^{1}\left(P_{\Omega}\right)$ implies the regularity $(\mathbf{u}, \tilde{p}) \in H^{2}\left(P_{\Omega}\right)^{d} \times H^{1}\left(P_{\Omega}\right)$ with the estimate

$$
\|\mathbf{u}\|_{H^{2}\left(P_{\Omega}\right)}+\|\tilde{p}\|_{H^{1}\left(P_{\Omega}\right)} \leq C_{\mathrm{S}}\left(\|\mathbf{f}\|_{L^{2}(\Omega)^{d}}+\|g\|_{H^{1}\left(P_{\Omega}\right)}\right) .
$$

The positive constant $C_{\mathrm{S}}>0$ is clearly independent of $\lambda$ since problem (6) does not depend on this parameter. An immediate consequence of (7) is that

$$
\|\mathbf{u}\|_{H^{2}\left(P_{\Omega}\right)}+\|\tilde{p}\|_{H^{1}\left(P_{\Omega}\right)} \leq C_{\mathrm{S}}\left(\|\mathbf{f}\|_{L^{2}(\Omega)^{d}}+\|\operatorname{div} \mathbf{u}\|_{H^{1}\left(P_{\Omega}\right)}\right) .
$$

Note that the optimal regularity shift is satisfies if for each corner of $P_{\Omega}$ (namely a common corner of some $\left.\Omega_{i}\right)$, there is no singular exponent in the strip $(0,1]$. We refer to [10, 11] for the standard Stokes system and to [12, 13, 5, for the extension to transmission Stokes problem. This condition can be interpreted as a geometrical one in the sense that the singular exponent depends on the values of $\mu$ and the angle of the subdomains $\Omega_{i}$ near the corner. In particular, if $\mu$ is constant in the whole domain, this optimal regularity shift holds if $\Omega$ is convex. Similarly, the assumption $\mathbf{u} \in H^{2}\left(P_{\Omega}\right)^{d}$ is related to the optimal regularity shift for the elastic transmission problem (3), we again refer to [10, 11, 14, 13, 5].

Theorem 2 (Regularity). There holds with $\mathcal{N}_{\mathbf{u}}$ defined by (2), assuming (8) and provided $\underline{\lambda}>C_{\mathrm{S}}$,

$$
\mathcal{N}_{\mathbf{u}} \leq C_{\underline{\lambda}, \mu}\|\mathbf{f}\|_{L^{2}(\Omega)^{d}},
$$

with $C_{\underline{\lambda}, \mu}$ dependent on $\Omega, \underline{\lambda}$, and $\mu$ but not on $\bar{\lambda}$.

Proof. By (8), there holds with $\tilde{p}$ defined by (5),

$$
\begin{aligned}
|\lambda \operatorname{div} \mathbf{u}|_{H^{1}\left(P_{\Omega}\right)} & =|\tilde{p}|_{H^{1}\left(P_{\Omega}\right)} \leq C_{\mathrm{S}}\left(\|\mathbf{f}\|_{L^{2}(\Omega)^{d}}+\|\operatorname{div} \mathbf{u}\|_{H^{1}\left(P_{\Omega}\right)}\right) \\
& \leq \frac{C_{\mathrm{S}}}{\underline{\lambda}^{1 / 2}}\left(\left\|\lambda^{1 / 2} \operatorname{div} \mathbf{u}\right\|_{L^{2}(\Omega)}+\frac{1}{\underline{\lambda}^{1 / 2}}|\lambda \operatorname{div} \mathbf{u}|_{H^{1}\left(P_{\Omega}\right)}\right)+C_{\mathrm{S}}\|\mathbf{f}\|_{L^{2}(\Omega)^{d}} .
\end{aligned}
$$


Hence, using the energy estimate (4),

$$
\left(1-\frac{C_{\mathrm{S}}}{\underline{\lambda}}\right)|\lambda \operatorname{div} \mathbf{u}|_{H^{1}\left(P_{\Omega}\right)} \leq C_{\mathrm{S}}\|\mathbf{f}\|_{L^{2}(\Omega)^{d}}\left(1+\frac{C_{\Omega}}{(\underline{\mu} \underline{\lambda})^{1 / 2}}\right),
$$

and the result follows from the assumption $\underline{\lambda}>C_{\mathrm{S}}$.

\section{Discrete setting}

\subsection{Notation}

Let $\mathcal{H} \subset \mathbb{R}_{*}^{+}$be a countable set of mesh sizes having 0 as its unique accumulation point, and denote by $\left(\mathcal{T}_{h}\right)_{h \in \mathcal{H}}$ a refined sequence of matching simplicial meshes $\mathcal{T}_{h}=\{T\}$ of $\Omega$. Let $h \in \mathcal{H}$ be arbitrary. The diameter of an element $T \in \mathcal{T}_{h}$ is denoted by $h_{T}$ and the mesh index is such that $h=\max _{T \in \mathcal{T}_{h}} h_{T}$. The set of faces of $\mathcal{T}_{h}$ is denoted by $\mathcal{F}_{h}$; boundary faces are collected in the set $\mathcal{F}_{h}^{\mathrm{b}}$ and we set $\mathcal{F}_{h}^{\mathrm{i}}:=\mathcal{F}_{h} \backslash \mathcal{F}_{h}^{\mathrm{b}}$. For all $F \in \mathcal{F}_{h}$ we let $\mathcal{T}_{F}:=\left\{T \in \mathcal{T}_{h} \mid F \subset \partial T\right\}$. For every interface $F \in \mathcal{F}_{h}^{\mathrm{i}}$, we select an arbitrary but fixed orientation of the normal $\mathbf{n}_{F}$ and number the elements of $\mathcal{T}_{F}$ in such a way that $\mathbf{n}_{F}$ points out of $T_{1}$; on boundary faces $F \in \mathcal{F}_{h}^{\mathrm{b}}$ the normal $\mathbf{n}_{F}$ is outward to $\Omega$. For all $F \in \mathcal{F}_{h}$ we denote by $h_{F}$ its diameter.

It is assumed in what follows that the mesh sequence $\left\{\mathcal{T}_{h}\right\}_{h \in \mathcal{H}}$ is shape-regular in the usual sense of Ciarlet [15], meaning that there exists $\rho>0$ such that

$$
\max _{h \in \mathcal{H}} \max _{T \in \mathcal{T}_{h}} \frac{h_{T}}{r_{T}} \leq \rho,
$$

where, for all $T \in \mathcal{T}_{h}, h \in \mathcal{H}, r_{T}$ denotes the radius of the largest ball inscribed in $T$. For all $h \in \mathcal{H}$ and all integers $k \geq 0$ we introduce the broken polynomial spaces:

$$
\mathbb{P}_{d}^{k}\left(\mathcal{T}_{h}\right):=\left\{v_{h} \in L^{2}(\Omega) \mid v_{h \mid T} \in \mathbb{P}_{d}^{k}(T), \forall T \in \mathcal{T}_{h}\right\},
$$

where $\mathbb{P}_{d}^{k}(T)$ denotes the restriction to $T$ of the polynomials of degree $\leq k$ in dimension $d$. Similarly, broken Sobolev spaces are defined for an integer $m \geq 0$ as

$$
H^{m}\left(\mathcal{T}_{h}\right):=\left\{v \in L^{2}(\Omega) \mid v_{\mid T} \in H^{m}(T), \forall T \in \mathcal{T}_{h}\right\} .
$$

The broken gradient acting on functions in $H^{1}\left(\mathcal{T}_{h}\right)$ is denoted by $\nabla_{h}$, the broken divergence acting on functions in $H^{1}\left(\mathcal{T}_{h}\right)^{d}$ is denoted by $\operatorname{div}_{h}$. Similarly, the broken versions of the symmetric gradient and elasticity operators $\underline{\underline{\epsilon}}$ and $\underline{\underline{\sigma}}$ are defined for all $\mathbf{v} \in H^{1}\left(\mathcal{T}_{h}\right)^{d}$ by setting

$$
\underline{\underline{\sigma_{h}}}(\mathbf{v}):=2 \mu \underline{\underline{\epsilon_{h}}}(\mathbf{v})+\lambda \operatorname{div}_{h} \mathbf{v} \underline{\underline{I_{d}}}, \quad \underline{\underline{\epsilon_{h}}}(\mathbf{v}):=\frac{1}{2}\left(\nabla_{h} \mathbf{v}+\nabla_{h} \mathbf{v}^{t}\right) .
$$

It is assumed here that, for all $h \in \mathcal{H}, \mathcal{T}_{h}$ is compatible with the partition $P_{\Omega}$, meaning that for all $T \in \mathcal{T}_{h}$ there exists a unique $\Omega_{i}, 1 \leq i \leq N_{\Omega}$, such that $T \subset \Omega_{i}$. This implies, in particular, that for all $h \in \mathcal{H}$,

$$
\mu \in \mathbb{P}_{d}^{0}\left(\mathcal{T}_{h}\right), \quad \lambda \in \mathbb{P}_{d}^{0}\left(\mathcal{T}_{h}\right) .
$$

We close this section by defining some trace operators commonly used in the context of $\mathrm{dG}$ methods. More precisely, for any scalar-valued function $v$ defined on $\Omega$ and smooth enough to admit on all $F \in \mathcal{F}_{h}$ a possibly two-valued trace on $F$ we let for all $F \in \mathcal{F}_{h}^{\mathrm{i}}$,

$$
\llbracket v \rrbracket(\mathbf{x}):=v_{\mid T_{1}}(\mathbf{x})-v_{\mid T_{2}}(\mathbf{x}), \quad\{v\}(\mathbf{x}):=\frac{1}{2}\left(v_{\mid T_{1}}(\mathbf{x})+v_{\mid T_{2}}(\mathbf{x})\right) .
$$

For all $F \in \mathcal{F}_{h}^{\mathrm{b}}$ such that $F=\partial T \cap \partial \Omega$ we conventionally set $\{v\}(\mathbf{x})=\llbracket v \rrbracket(\mathbf{x})=v_{\mid T}(\mathbf{x})$. When applied to vector- or tensor-valued functions, the jump and average operators act component-wise. If no confusion can arise, the variable $\mathbf{x}$ is omitted, and we simply write $\{v\}$ and $\llbracket v \rrbracket$. 


\subsection{Preliminary results}

In this section we recall some preliminary results, namely the discrete Korn inequality in broken polynomial spaces and the definition of jump liftings.

\subsubsection{Discrete Korn's inequality}

We define the following usual $H_{0}^{1}$-like norm on $H_{0}^{1}\left(\mathcal{T}_{h}\right)$ :

$$
\|v\|_{1, h}^{2}:=\left\|\nabla_{h} v\right\|_{L^{2}(\Omega)^{d}}^{2}+|v|_{\mathrm{J}}^{2}, \quad\left|v_{h}\right|_{\mathrm{J}}^{2}:=\sum_{F \in \mathcal{F}_{h}} \frac{1}{h_{F}}\left\|\llbracket v_{h} \rrbracket\right\|_{L^{2}(F)}^{2} .
$$

An important ingredient in the approximation of the linear elasticity problem is the discrete counterpart of Korn's inequality, which states that the $\|\cdot\|_{1, h}$-norm can be controlled in terms of the $L^{2}$-norm of the symmetric part of the gradient plus the jump seminorm $|\cdot|_{\mathrm{J}}$. Korn's inequalities for piecewise $H^{1}$ functions on fairly general meshes are proved by Brenner [6]. In this work we make use of the following variant of [6, (1.11)].

Theorem 3 (Discrete Korn's inequality). There is $C_{\mathrm{K}}$ uniquely depending on the mesh regularity parameter and on $\Omega$ such that, for all $\mathbf{v}_{h} \in \mathbb{P}_{d}^{k}\left(\mathcal{T}_{h}\right)^{d}, k \geq 1$,

$$
\left\|\mathbf{v}_{h}\right\|_{1, h} \leq C_{\mathrm{K}}\left(\left\|\underline{\underline{\epsilon}}_{h}\left(\mathbf{v}_{h}\right)\right\|_{L^{2}(\Omega)^{d, d}}^{2}+\left|\mathbf{v}_{h}\right|_{\mathrm{J}}^{2}\right)^{1 / 2}
$$

\subsubsection{Liftings}

For an integer polynomial degree $l \geq 0$ we define a face lifting operator inspired by Brezzi e.a. [16] as follows: For all $F \in \mathcal{F}_{h}$ and all $\mathbf{v} \in L^{2}(F)^{d}, \underline{r_{F}^{l}}(\mathbf{v}) \in \mathbb{P}_{d}^{l}\left(\mathcal{T}_{h}\right)^{d, d}$ is the unique solution to

$$
\int_{\Omega} \underline{\underline{r_{F}^{l}}}(\mathbf{v}): \underline{\underline{\tau_{h}}}=\int_{F} \mathbf{v} \otimes \mathbf{n}_{F}:\left\{\underline{\underline{\tau_{h}}}\right\} \quad \forall \underline{\underline{\tau_{h}}} \in \mathbb{P}_{d}^{l}\left(\mathcal{T}_{h}\right)^{d, d},
$$

where, for two vectors $\mathbf{a}$ and $\mathbf{b}$ we have let $\mathbf{a} \otimes \mathbf{b}:=\left[a_{i} b_{j}\right]_{1 \leq i, j \leq d} \in \mathbb{R}^{d, d}$. For the sake of brevity we also introduce a symbol for the trace of the face lifting,

$$
r_{F}^{l}(\mathbf{v}):=\operatorname{tr}\left(\underline{\underline{r_{F}^{l}}}(\mathbf{v})\right) \in \mathbb{P}_{d}^{l}\left(\mathcal{T}_{h}\right)
$$

The piecewise constant liftings obtained by taking $l=0$ can be related to the average of $\mathbf{v}$ across one face. More precisely, taking $\underline{\underline{\tau_{h}}}=\chi_{T} \underline{\underline{m_{i j}}}$ for $1 \leq i, j \leq d$ and $T \in \mathcal{T}_{F}$ in 10 where $\left(\underline{\underline{m_{i j}}}\right)_{i^{\prime} j^{\prime}}=\delta_{i i^{\prime}} \delta_{j j^{\prime}}$ and $\chi_{T}$ denotes the characteristic function of $T$, it is inferred

$$
\underline{\underline{r_{F}^{0}}}(\mathbf{v})_{\mid T} \equiv \frac{|F|_{d-1}}{\operatorname{card}\left(\mathcal{T}_{F}\right)|T|_{d}}\langle\mathbf{v}\rangle_{F} \otimes \mathbf{n}_{F}, \quad r_{F}^{0}(\mathbf{v})_{\mid T} \equiv \frac{|F|_{d-1}}{\operatorname{card}\left(\mathcal{T}_{F}\right)|T|_{d}}\langle\mathbf{v}\rangle_{F} \cdot \mathbf{n}_{F}
$$

where, for all functions $\varphi$ integrable on $F$, we have let $\langle\varphi\rangle_{F}:=\int_{F} \varphi /|F|_{d-1}$. The relations (11) can be replace the liftings in practical implementations. Finally, for a function $\mathbf{v} \in H_{0}^{1}(\Omega)^{d}$, the global lifting of the jumps of $\mathbf{v}$ and its trace are respectively denoted by

$$
\left.\underline{\underline{R_{h}^{l}}}(\mathbf{v}):=\sum_{F \in \mathcal{F}_{h}} \underline{\underline{r_{F}^{l}}}(\llbracket \mathbf{v} \rrbracket) \in \mathbb{P}_{d}^{l}\left(\mathcal{T}_{h}\right)^{d, d}, \quad R_{h}^{l}(\mathbf{v}):=\operatorname{tr} \underline{\underline{R_{h}^{l}}}(\mathbf{v})\right)=\sum_{F \in \mathcal{F}_{h}} r_{F}^{l}(\llbracket \mathbf{v} \rrbracket) \in \mathbb{P}_{d}^{l}\left(\mathcal{T}_{h}\right) .
$$

\subsection{The discrete problem}

We introduce the following spaces:

$$
\mathbf{U}_{h}:=\mathbb{P}_{d}^{1}\left(\mathcal{T}_{h}\right)^{d}, \quad \mathbf{U}_{*}:=\mathbf{U} \cap H^{2}\left(P_{\Omega}\right)^{d} \quad \mathbf{U}_{* h}:=\mathbf{U}_{*}+\mathbf{U}_{h} .
$$


The additional regularity in $\mathbf{U}_{*}$ ensures that the traces of gradients on mesh faces are squareintegrable. This regularity assumption can be relaxed using the techniques of [17, To which we refer for further details. We define the discrete bilinear form $a_{h} \in \mathcal{L}\left(\mathbf{U}_{* h} \times \mathbf{U}_{* h}, \mathbb{R}\right)$ such that

$$
\begin{aligned}
a_{h}(\mathbf{w}, \mathbf{v}):= & \int_{\Omega} \underline{\underline{\sigma_{h}}}(\mathbf{w}): \underline{\underline{\underline{\epsilon_{h}}}}(\mathbf{v}) \\
& -\sum_{F \in \mathcal{F}_{h}} \int_{F}\left(\left\{\underline{\underline{\sigma_{h}}}(\mathbf{w})\right\}:\langle\llbracket \mathbf{v} \rrbracket\rangle_{F} \otimes \mathbf{n}_{F}+\langle\llbracket \mathbf{w} \rrbracket\rangle_{F} \otimes \mathbf{n}_{F}:\left\{\underline{\underline{\sigma_{h}}}(\mathbf{v})\right\}\right) \\
& +\sum_{F \in \mathcal{F}_{h}} \int_{\Omega} \eta\left(2 \mu \underline{\underline{r_{F}^{0}}}(\llbracket \mathbf{w} \rrbracket): \underline{\underline{r_{F}^{0}}}(\llbracket \mathbf{v} \rrbracket)+\lambda r_{F}^{0}(\llbracket \mathbf{w} \rrbracket) r_{F}^{0}(\llbracket \mathbf{v} \rrbracket)\right) \\
& +\sum_{F \in \mathcal{F}_{h}} \int_{F} \frac{\gamma_{\mu, F}}{h_{F}} \llbracket \mathbf{w} \rrbracket \cdot \llbracket \mathbf{v} \rrbracket,
\end{aligned}
$$

where $\eta>0$ denotes a user-dependent positive parameter and, for all $F \in \mathcal{F}_{h}, \gamma_{\mu, F}:=\max _{T \in \mathcal{T}_{F}} \mu_{\mid T}$. The discrete problem reads

$$
\text { Find } \mathbf{u}_{h} \in \mathbf{U}_{h} \text { s.t. } a_{h}\left(\mathbf{u}_{h}, \mathbf{v}_{h}\right)=\int_{\Omega} \mathbf{f} \cdot \mathbf{v}_{h} \text { for all } \mathbf{v}_{h} \in \mathbf{U}_{h} .
$$

The terms in the second line of 12 are responsible, respectively, for the (weak) consistency and symmetry of the bilinear form $a_{h}$; the terms in the third line ensure nonnegativity by penalizing the jump liftings across mesh faces to compensate the corresponding contribution from the weak consistency and symmetry terms. Using (11), it is a simple matter to realize that these contributions only penalize the mean value of the jumps; finally, in view of the discrete Korn inequality $(9)$, the term in the fourth line contains a full penalization of jumps to ensure coercivity.

Remark 4 (Lifting-based penalty terms). Considering lifting-based penalty terms in the third line of (12) allows to derive a trivial lower bound for the user-dependent parameter $\eta$ to achieve stability (cf. Lemma 7). In practice, the following equivalent expression based on (11) can be used:

$$
\sum_{F \in \mathcal{F}_{h}} \int_{F}\left(\eta_{\mu, F}\langle\mathbf{w}\rangle_{F} \cdot\langle\mathbf{v}\rangle_{F}+\eta_{\lambda, F}\left(\langle\mathbf{w}\rangle_{F} \cdot \mathbf{n}_{F}\right)\left(\langle\mathbf{v}\rangle_{F} \cdot \mathbf{n}_{F}\right)\right)
$$

where $\eta_{\mu, F}:=\eta \sum_{T \in \mathcal{T}_{F}} \frac{(2 \mu)_{\mid T}|F|_{d-1}}{\operatorname{card}\left(\mathcal{T}_{F}\right)^{2}|T|_{d}}$ and $\eta_{\lambda, F}:=\eta \sum_{T \in \mathcal{T}_{F}} \frac{\lambda_{\mid T}|F|_{d-1}}{\operatorname{card}\left(\mathcal{T}_{F}\right)^{2}|T|_{d}}$.

Remark 5 (Restriction of $a_{h}$ to $\mathbf{U}_{h} \times \mathbf{U}_{h}$ and Crouzeix-Raviart finite elements). Using the fact that $\underline{\underline{\sigma_{h}}}\left(\mathbf{v}_{h}\right) \in \mathbb{P}_{d}^{0}\left(\mathcal{T}_{h}\right)^{d, d}$ for all $\mathbf{v}_{h} \in \mathbf{U}_{h}$, it is inferred that, for all $\left(\mathbf{w}_{h}, \mathbf{v}_{h}\right) \in \mathbf{U}_{h}^{2}$, the asymptotic consistency and symmetry terms in the second line of 12 are equivalent to

$$
-\sum_{F \in \mathcal{F}_{h}} \int_{F}\left(\left\{\underline{\underline{\sigma_{h}}}\left(\mathbf{w}_{h}\right)\right\}: \llbracket \mathbf{v}_{h} \rrbracket \otimes \mathbf{n}_{F}+\llbracket \mathbf{w}_{h} \rrbracket \otimes \mathbf{n}_{F}:\left\{\underline{\underline{\sigma_{h}}}\left(\mathbf{v}_{h}\right)\right\}\right) .
$$

Using this formulation to extend $a_{h}$ to $\mathbf{U}_{* h} \times \mathbf{U}_{* h}$ would yield a consistent bilinear form. However, we have preferred to use the asimptotically consistent formulation $(12)$ since it makes it easier to track the dependency on $\lambda$ and $\mu$ of the multiplicative parameter in the error estimate, as detailed in the following section. Moreover, as is the case in [3], the analysis readily applies when the dG space $\mathbf{U}_{h}$ is replaced by the Crouzeix-Raviart finite element space $\mathbb{C R}\left(\mathcal{T}_{h}\right)^{d}$ defined by $(16)$.

\subsection{Energy error estimate}

Lemma 6 (Weak consistency). Let $\mathbf{u} \in \mathbf{U}$ denote the solution to the continuous problem (1) and further assume that $\mathbf{u} \in \mathbf{U}_{*}$. Then,

$$
\forall \mathbf{v}_{h} \in \mathbf{U}_{h}, \quad a_{h}\left(\mathbf{u}, \mathbf{v}_{h}\right)=\int_{\Omega} \mathbf{f} \cdot \mathbf{v}_{h}-\mathcal{E}_{\mathbf{u}}\left(\mathbf{v}_{h}\right),
$$

with consistency error $\mathcal{E}_{\mathbf{u}}\left(\mathbf{v}_{h}\right):=\sum_{F \in \mathcal{F}_{h}} \int_{F}\{\underline{\underline{\sigma}}(\mathbf{u})\}:\left(\left\langle\llbracket \mathbf{v}_{h} \rrbracket\right\rangle_{F}-\llbracket \mathbf{v}_{h} \rrbracket\right) \otimes \mathbf{n}_{F}$. 
Proof. Using the symmetry of $\underline{\underline{\sigma}}(\mathbf{u})$, integrating by parts, and rearranging the boundary terms it is inferred for all $\mathbf{v}_{h} \in \mathbf{U}_{h}$,

$$
\int_{\Omega} \underline{\underline{\sigma}}(\mathbf{u}): \underline{\underline{\epsilon_{h}}}\left(\mathbf{v}_{h}\right)=\int_{\Omega} \mathbf{f} \cdot \mathbf{v}_{h}+\sum_{F \in \mathcal{F}_{h}} \int_{F}\{\underline{\underline{\sigma}}(\mathbf{u})\}: \llbracket \mathbf{v}_{h} \rrbracket \otimes \mathbf{n}_{F}+\sum_{F \in \mathcal{F}_{h}^{\mathrm{i}}} \int_{F} \llbracket \underline{\underline{\sigma}}(\mathbf{u}) \rrbracket:\left\{\mathbf{v}_{h}\right\} \otimes \mathbf{n}_{F} .
$$

The conclusion follows since $\llbracket \underline{\underline{\sigma}}(\mathbf{u}) \mathbf{n}_{F} \rrbracket_{F}=\mathbf{0}$ for all $F \in \mathcal{F}_{h}^{\mathrm{i}}$ and $\llbracket \mathbf{u} \rrbracket_{F}=\mathbf{0}$ for all $F \in \mathcal{F}_{h}$.

Stability is expressed with respect to the following energy-like norm defined on $H^{1}\left(\mathcal{T}_{h}\right)^{d}$ :

$$
\|\mathbf{v}\|_{\mu, \lambda}^{2}:=\left\|(2 \mu)^{1 / 2} \underline{\underline{\epsilon_{h}}}(\mathbf{v})\right\|_{L^{2}(\Omega)^{d, d}}^{2}+\left\|\lambda^{1 / 2} \operatorname{div}_{h} \mathbf{v}\right\|_{L^{2}(\Omega)}^{2}+|\mathbf{v}|_{\mathbf{r}, \mu}^{2}+|\mathbf{v}|_{\mathbf{r}, \lambda}^{2}+|\mathbf{v}|_{J, \mu}^{2}
$$

with seminorms $|\mathbf{v}|_{\mathbf{r}, \mu}^{2}:=\sum_{F \in \mathcal{F}_{h}}\left\|(2 \mu)^{1 / 2} \underline{\underline{r_{F}^{0}}}(\llbracket \mathbf{v} \rrbracket)\right\|_{L^{2}(\Omega)}^{2},|\mathbf{v}|_{\mathrm{r}, \lambda}^{2}:=\sum_{F \in \mathcal{F}_{h}}\left\|\lambda^{1 / 2} r_{F}^{0}(\llbracket \mathbf{v} \rrbracket)\right\|_{L^{2}(\Omega)}^{2}$, and $|\mathbf{v}|_{J, \mu}^{2}:=\sum_{F \in \mathcal{F}_{h}}\left\|\left(\gamma_{\mu, F} / h_{F}\right)^{1 / 2} \llbracket \mathbf{v} \rrbracket\right\|_{L^{2}(F)^{d}}^{2}$.

Lemma 7 (Coercivity). For all $\eta>N_{\partial}=d+1$ there holds with $\alpha_{\eta}:=\left(\eta-N_{\partial}\right) /(1+\eta)$,

$$
\forall \mathbf{v}_{h} \in \mathbf{U}_{h}, \quad a_{h}\left(\mathbf{v}_{h}, \mathbf{v}_{h}\right) \geq \alpha_{\eta}\left\|\mathbf{v}_{h}\right\|_{\mu, \lambda}^{2} \cdot \geq \alpha_{\eta} \underline{\mu}^{2} C_{\mathrm{K}}\left\|\mathbf{v}_{h}\right\|_{1, h}^{2},
$$

where $C_{\mathrm{K}}$ is the discrete Korn constant introduced in (9).

Proof. Taking $(\mathbf{w}, \mathbf{v})=\left(\mathbf{v}_{h}, \mathbf{v}_{h}\right)$ in $(12)$ it is a simple matter to realize that only the weak consistency and symmetry terms in the second line do not have a sign a priori. To bound these terms, we use the definition of the lifting operators given in Sect. 3.2.2 together with the Cauchy-Schwarz inequality to infer

$$
\begin{aligned}
\left|\sum_{F \in \mathcal{F}_{h}} \int_{F}\left\{\underline{\underline{\sigma_{h}}}\left(\mathbf{v}_{h}\right)\right\}:\left\langle\llbracket \mathbf{v}_{h} \rrbracket\right\rangle_{F} \otimes \mathbf{n}_{F}\right| & =\left|\int_{\Omega}\left(2 \mu \underline{\underline{\epsilon_{h}}}\left(\mathbf{v}_{h}\right)+\lambda \operatorname{div}_{h} \mathbf{v}_{h}\right): \underline{\underline{R_{h}^{0}}}\left(\mathbf{v}_{h}\right)\right| \\
& \left.\leq N_{\partial}\left(\left\|(2 \mu)^{1 / 2} \underline{\underline{\epsilon_{h}}}\left(\mathbf{v}_{h}\right)\right\|_{L^{2}(\Omega)}\right)^{d, d}\left|\mathbf{v}_{h}\right|_{\mathrm{r}, \mu}+\left\|\lambda^{1 / 2} \operatorname{div}_{h} \mathbf{v}_{h}\right\|_{L^{2}(\Omega)}\left|\mathbf{v}_{h}\right|_{\mathrm{r}, \lambda}\right)
\end{aligned}
$$

where we have used the fact that, for all $F \in \mathcal{F}_{h}$, the local lifting is supported in the elements of $\mathcal{T}_{F}$ to infer $\left\|(2 \mu)^{1 / 2} \underline{\underline{R_{h}^{l}}}\left(\mathbf{v}_{h}\right)\right\|_{L^{2}(\Omega)^{d, d}}^{2} \leq N_{\partial}\left|\mathbf{v}_{h}\right|_{\mathrm{r}, \mu}^{2}$ and $\left\|\lambda^{1 / 2} R_{h}^{l}\left(\mathbf{v}_{h}\right)\right\|_{L^{2}(\Omega)}^{2} \leq N_{\partial}\left|\mathbf{v}_{h}\right|_{\mathrm{r}, \lambda}^{2}$ (cf., e.g., [18,

Lemma 4.34] for details). The conclusion follows using twice the inequality $x^{2}-2 N_{\partial}^{1 / 2} x y+\eta y^{2} \geq$ $\frac{\eta-N_{\partial}}{1+\eta}\left(x^{2}+y^{2}\right)$ with (i) $x=\left\|(2 \mu)^{1 / 2} \underline{\underline{\epsilon_{h}}}\left(\mathbf{v}_{h}\right)\right\|_{L^{2}(\Omega)^{d, d}}$ and $y=\left|\mathbf{v}_{h}\right|_{\mathbf{r}, \mu} ;\left(\right.$ ii) $x=\left\|\lambda^{1 / 2} \operatorname{div}_{h} \mathbf{v}_{h}\right\|_{L^{2}(\Omega)}$ and $y=\left|\mathbf{v}_{h}\right|_{\mathrm{r}, \lambda}$.

The last ingredient to prove an error estimate is to show the continuity of $a_{h}$ in $\mathbf{U}_{* h} \times \mathbf{U}_{h}$. To this end, we define an augmented version of the energy norm on $H^{1}\left(\mathcal{T}_{h}\right)^{d}$ as follows:

$$
\|\mathbf{v}\|_{\mu, \lambda, *}^{2}:=\|\mathbf{v}\|_{\mu, \lambda}^{2}+\sum_{T \in \mathcal{T}_{h}} h_{T}\left(\left\|\left((2 \mu)^{1 / 2} \underline{\underline{\epsilon_{h}}}\left(\mathbf{v}_{h}\right)\right)_{\mid T}\right\|_{L^{2}(\partial T)^{d, d}}^{2}+\left\|\left(\lambda^{1 / 2} \operatorname{div}_{h} \mathbf{v}_{h}\right)_{\mid T}\right\|_{L^{2}(\partial T)}^{2}\right) .
$$

Clearly, $\|\mathbf{v}\|_{\mu, \lambda, *} \geq\|\mathbf{v}\|_{\mu, \lambda}$ for all $\mathbf{v} \in H^{1}\left(\mathcal{T}_{h}\right)^{d}$, and the two norms are uniformly equivalent on $\mathbf{U}_{h}$.

Lemma 8 (Boundedness). There holds with $\beta_{\rho, \eta}:=2+\eta+2 \rho^{\frac{1}{2}}$,

$$
\forall\left(\mathbf{w}, \mathbf{v}_{h}\right) \in \mathbf{U}_{* h} \times \mathbf{U}_{h}, \quad a_{h}\left(\mathbf{w}, \mathbf{v}_{h}\right) \leq \beta_{\rho, \eta}\|\mathbf{w}\|_{\mu, \lambda, *}\left\|\mathbf{v}_{h}\right\|_{\mu, \lambda} .
$$

Proof. Denote by $\mathfrak{T}_{1}, \ldots, \mathfrak{T}_{6}$ the addends in the right-hand side of 12 with $(\mathbf{w}, \mathbf{v})=\left(\mathbf{w}, \mathbf{v}_{h}\right)$. Multiple applications of the Cauchy-Schwarz inequality yield

$$
\left|\mathfrak{T}_{1}\right|+\left|\mathfrak{T}_{4}\right|+\left|\mathfrak{T}_{5}\right|+\left|\mathfrak{T}_{6}\right| \leq(1+\eta)\|\mathbf{w}\|_{\mu, \lambda}\left\|\mathbf{v}_{h}\right\|_{\mu, \lambda} \leq(1+\eta)\|\mathbf{w}\|_{\mu, \lambda, *}\left\|\mathbf{v}_{h}\right\|_{\mu, \lambda} .
$$


Using the equivalent form $\mathfrak{T}_{3}=\sum_{F \in \mathcal{F}_{h}} \int_{F} \underline{\underline{r_{F}^{0}}}(\llbracket \mathbf{w} \rrbracket): \underline{\underline{\sigma_{h}}}\left(\mathbf{v}_{h}\right)$, an application of the Cauchy-Schwarz inequality readily yields $\left|\mathfrak{T}_{3}\right| \leq\|\mathbf{w}\|_{\mu, \lambda, *}\left\|\mathbf{v}_{h}\right\|_{\mu, \lambda}$. Finally, to estimate $\mathfrak{T}_{2}$ we use the CauchySchwarz inequality to infer

$$
\left|\mathfrak{T}_{2}\right| \leq\|\mathbf{w}\|_{\mu, \lambda, *} \times\left\{\sum_{F \in \mathcal{F}_{h}} \frac{1}{h_{F}} \sum_{T \in \mathcal{T}_{F}}\left(\left\|\left(2 \mu_{\mid T}\right)^{1 / 2}\left\langle\llbracket \mathbf{v}_{h} \rrbracket\right\rangle_{F}\right\|_{L^{2}(F), d, d}^{2}+\left\|\lambda_{\mid T}^{1 / 2}\left\langle\llbracket \mathbf{v}_{h} \rrbracket\right\rangle_{F} \cdot \mathbf{n}_{F}\right\|_{L^{2}(F)}^{2}\right)\right\}^{\frac{1}{2}} .
$$

Using (11) and since, owing to mesh regularity, for all $T \in \mathcal{T}_{h}$ and all $F \in \mathcal{F}_{T}$, there holds $\frac{\operatorname{card}\left(\mathcal{T}_{F}\right)^{2}|T|_{d}}{h_{F}|F|_{d-1}} \leq 4 \rho$, the term in braces is bounded by $4 \rho\left(\left|\mathbf{v}_{h}\right|_{\mathbf{r}, \mu}^{2}+\left|\mathbf{v}_{h}\right|_{\mathrm{r}, \lambda}^{2}\right)$. This concludes the proof.

The following result is a classical consequence of Lemmata 6,7 and 8 .

Lemma $\mathbf{9}$ (Error estimate). Let $\mathbf{u}$ denote the unique solution to (3) and further assume $\mathbf{u} \in \mathbf{U}_{*}$. Then, denoting by $\mathbf{u}_{h}$ the unique solution to (13), there holds

$$
\left\|\mathbf{u}-\mathbf{u}_{h}\right\|_{\mu, \lambda} \leq\left(1+\frac{\beta_{\rho, \eta}}{\alpha_{\eta}}\right) \inf _{\mathbf{v}_{h} \in \mathbf{U}_{h}}\left\|\mathbf{u}-\mathbf{v}_{h}\right\|_{\mu, \lambda, *}+\sup _{\mathbf{v}_{h} \in \mathbf{U}_{h}} \frac{\mathcal{E}_{\mathbf{u}}\left(\mathbf{v}_{h}\right)}{\left\|\mathbf{v}_{h}\right\|_{\mu, \lambda}} .
$$

Following Hansbo and Larson [3, we derive an estimate for the convergence rate using the Crouzeix-Raviart interpolator $\mathcal{I}_{\mathbb{C R}}: H^{2}\left(\mathcal{T}_{h}\right) \rightarrow \mathbb{C R}\left(\mathcal{T}_{h}\right)$, where

$$
\mathbb{C R}\left(\mathcal{T}_{h}\right):=\left\{v_{h} \in \mathbb{P}_{d}^{1}\left(\mathcal{T}_{h}\right) \mid\left\langle\llbracket v_{h} \rrbracket\right\rangle_{F}=0, \forall F \in \mathcal{F}_{h}\right\}
$$

When applied to vector functions, the interpolator acts componentwise. The following approximation estimates classically hold (cf. [3, Lemma 2.3] and references therein): For all $\mathbf{v} \in H^{2}\left(\mathcal{T}_{h}\right)^{d}$ and all $T \in \mathcal{T}_{h}$

$$
\begin{aligned}
\left\|\mathbf{v}-\mathcal{I}_{\mathbb{C R}} \mathbf{v}\right\|_{L^{2}(T)^{d}}+h_{T}\left|\mathbf{v}-\mathcal{I}_{\mathbb{C R}} \mathbf{v}\right|_{H^{1}(T)^{d}} & \leq C_{\mathbb{C R}} h_{T}^{2}\|\mathbf{v}\|_{H^{2}(T)^{d}}, \\
\left\|\operatorname{div}\left(\mathbf{v}-\mathcal{I}_{\mathbb{C R}} \mathbf{v}\right)\right\|_{L^{2}(T)}+h_{T}\left|\operatorname{div}\left(\mathbf{v}-\mathcal{I}_{\mathbb{C R}} \mathbf{v}\right)\right|_{H^{1}(T)} & \leq C_{\mathbb{C R}} h_{T}|\operatorname{div} \mathbf{v}|_{H^{1}(T)},
\end{aligned}
$$

where $C_{\mathbb{C R}}$ only depends on the mesh regularity parameter $\rho$. Observe that the broken $H^{1}$ seminorm of the divergence appears in the right-hand side of $17 \mathrm{~b}$ coherently with the definition of $\mathcal{N}_{\mathbf{u}}$ (2). Indeed, (17b) results from a simple application of the Poincaré-Wirtinger inequality observing that $\operatorname{div}\left(\mathcal{I}_{\mathbb{C R}} \mathbf{v}\right)=\langle\operatorname{div} \mathbf{v}\rangle_{\Omega}$. This is an important property meaning that the discrete space allows to accurately approximate nontrivial functions with zero divergence.

Theorem 10 (Convergence rate). There holds

$$
\left\|\mathbf{u}-\mathbf{u}_{h}\right\|_{\mu, \lambda} \leq \chi h,
$$

with $\chi:=C_{\rho, \mu} \mathcal{N}_{\mathbf{u}}$ where $\mathcal{N}_{\mathbf{u}}$ is defined by (2) and $C_{\rho, \mu}$ only depends on the mesh regularity parameter $\rho$ and on $\mu$.

Proof. For the sake of brevity we denote by $a \lesssim b$ the inequality $a \leq C b$ with $C$ generic constant only depending on the mesh regularity parameter $\rho$ and on $\mu$.

(i) Approximation error. Let $\mathbf{w}_{h}:=\mathcal{I}_{\mathbb{C R}} \mathbf{u}$. The fact that $\left\|\mathbf{u}-\mathbf{w}_{h}\right\|_{\mu, \lambda, *} \lesssim \mathcal{N}_{\mathbf{u}} h$ follows from (17) upon observing that $\left|\mathbf{u}-\mathbf{w}_{h}\right|_{\mathrm{r}, \mu}=\left|\mathbf{u}-\mathbf{w}_{h}\right|_{\mathrm{r}, \lambda}=0$ and applying several times the following trace inequality (see, e.g., Monk and Süli [19] or Carstensen and Funken [20]): For all $v \in H^{1}\left(\mathcal{T}_{h}\right)$,

$$
\forall T \in \mathcal{T}_{h}, \quad h_{T}\|v\|_{L^{2}(\partial T)}^{2} \lesssim\|v\|_{L^{2}(T)}^{2}+h_{T}^{2}\|\nabla v\|_{L^{2}(T)^{d}}^{2}
$$

(ii) Consistency error. Let $\mathbf{v}_{h} \in \mathbf{U}_{h}$ and denote by $\pi_{h}^{0}$ the $L^{2}$-orthogonal projector onto $\mathbb{P}_{d}^{0}\left(\mathcal{T}_{h}\right)^{d, d}$. 
Using the fact that $\left\{\pi_{h}^{0} \underline{\underline{\sigma}}(\mathbf{u})\right\}_{F}$ is constant over $F \in \mathcal{F}_{h}$ together with the Cauchy-Schwarz inequality, it is inferred

$$
\begin{aligned}
\mathcal{E}_{\mathbf{u}}\left(\mathbf{v}_{h}\right)= & \sum_{F \in \mathcal{F}_{h}} \int_{F}\left\{\underline{\underline{\sigma}}(\mathbf{u})-\pi_{h}^{0} \underline{\underline{\sigma}}(\mathbf{u})\right\}:\left(\left\langle\llbracket \mathbf{v}_{h} \rrbracket\right\rangle_{F}-\llbracket \mathbf{v}_{h} \rrbracket\right) \\
\lesssim & \left\{\sum_{T \in \mathcal{T}_{h}} h_{T}\left(\left\|\left(2 \mu\left(\underline{\underline{\epsilon}}(\mathbf{u})-\pi_{h}^{0} \underline{\underline{\epsilon}}(\mathbf{u})\right)\right)_{\mid T}\right\|_{L^{2}(\partial T)^{d, d}}^{2}+\left\|\left(\lambda\left(\operatorname{div} \mathbf{u}-\pi_{h}^{0} \operatorname{div} \mathbf{u}\right)\right)_{\mid T}\right\|_{L^{2}(\partial T)}^{2}\right)\right\}^{\frac{1}{2}} \\
& \times\left\{\sum_{F \in \mathcal{F}_{h}} h_{F}^{-1}\left\|\left\langle\llbracket \mathbf{v}_{h} \rrbracket\right\rangle_{F}-\llbracket \mathbf{v}_{h} \rrbracket\right\|_{L^{2}(F)^{d}}^{2}\right\}^{\frac{1}{2}}:=\mathfrak{T}_{1} \times \mathfrak{T}_{2} .
\end{aligned}
$$

Using (19), it is readily inferred that $\mathfrak{T}_{1} \lesssim \mathcal{N}_{\mathbf{u}} h$. On the other hand, the generalized PoincaréFriedrichs inequality $\|v-\llbracket v \rrbracket\|_{L^{2}(F)} \lesssim h_{F}^{1 / 2}\|\nabla v\|_{L^{2}\left(\mathcal{T}_{F}\right)^{d}}$ valid for all $v \in H^{1}\left(\mathcal{T}_{h}\right) \cap H_{0}^{1}(\Omega)$ (cf. [21]) yields $\mathfrak{T}_{2} \lesssim\left\|\nabla \mathbf{v}_{h}\right\|_{L^{2}(\Omega)^{d, d}}$. Finally, using $(9), \mathfrak{T}_{2} \lesssim\left\|\mathbf{v}_{h}\right\|_{\mu, \lambda}$, and the conclusion follows.

Remark 11 (Numerical locking). The estimate (18) shows that the discrete method (13) is lockingfree for $d=2$, since, owing to Theorem 2 , the multiplicative parameter $\chi$ only depends on quantities that stay bounded for $\bar{\lambda} \rightarrow+\infty$.

\subsection{Flux formulation}

It is a common practice to express dG methods in terms of numerical fluxes. To this purpose, we introduce the following lifting-corrected gradient: For all $\mathbf{v}_{h} \in \mathbf{U}_{h}$,

$$
\underline{\underline{G_{h}^{0}}}\left(\mathbf{v}_{h}\right):=\nabla_{h} \mathbf{v}_{h}-\underline{\underline{R_{h}^{0}}}\left(\mathbf{v}_{h}\right) .
$$

Discrete symmetric gradient, divergence, and elasticity operators can be defined from $\underline{\underline{G_{h}^{0}}}\left(\mathbf{v}_{h}\right)$ :

$$
\begin{aligned}
& \underline{\underline{E_{h}^{0}}}\left(\mathbf{v}_{h}\right):=\frac{1}{2}\left(\underline{\underline{G_{h}^{0}}}\left(\mathbf{v}_{h}\right)+\underline{\underline{G_{h}^{0}}}\left(\mathbf{v}_{h}\right)^{t}\right) \\
& D_{h}^{0}\left(\mathbf{v}_{h}\right):=\operatorname{tr}\left(\underline{\underline{G_{h}^{0}}}\left(\mathbf{v}_{h}\right)\right)=\operatorname{div}_{h} \mathbf{v}_{h}-R_{h}^{0}\left(\mathbf{v}_{h}\right) \\
& \underline{\underline{\Sigma_{h}^{0}}}\left(\mathbf{v}_{h}\right):=2 \mu \underline{\underline{E_{h}^{0}}}\left(\mathbf{v}_{h}\right)+\lambda D_{h}^{0}\left(\mathbf{v}_{h}\right) \underline{\underline{I_{d}}}
\end{aligned}
$$

Lemma 12 (Flux formulation). There holds for all $\left(\mathbf{w}_{h}, \mathbf{v}_{h}\right) \in \mathbf{U}_{h}^{2}$,

$$
a_{h}\left(\mathbf{w}_{h}, \mathbf{v}_{h}\right)=\int_{\Omega} \underline{\underline{\Sigma_{h}^{0}}}\left(\mathbf{w}_{h}\right): \underline{\underline{\underline{h}}}\left(\mathbf{v}_{h}\right)+\sum_{F \in \mathcal{F}_{h}} \int_{F} \underline{\underline{\Phi_{F}}}\left(\mathbf{w}_{h}\right): \llbracket \mathbf{v}_{h} \rrbracket \otimes \mathbf{n}_{F}
$$

where

$$
\underline{\underline{\Phi_{F}}}\left(\mathbf{w}_{h}\right)=-\left\{2 \mu\left(\underline{\underline{\epsilon_{h}}}\left(\mathbf{w}_{h}\right)-\underline{\underline{r_{F}^{0}}}\left(\mathbf{w}_{h}\right)\right)+\lambda\left(\operatorname{div}_{h} \mathbf{w}_{h}-\underline{\underline{r_{F}^{0}}}\left(\mathbf{w}_{h}\right)\right) \underline{\underline{I_{d}}}\right\}+\frac{\gamma_{\mu, F}}{h_{F}} \llbracket \mathbf{w}_{h} \rrbracket \otimes \mathbf{n}_{F} .
$$

\subsection{Convergence to minimal regularity solutions}

For the sake of completeness, this section briefly addresses the convergence to minimal regularity solutions, i.e., solutions that barely sit in $H_{0}^{1}(\Omega)^{d}$. In view of applying the arguments of [22], we record the following equivalent expression for the bilinear form $a_{h}$ with discrete arguments: For all $\left(\mathbf{w}_{h}, \mathbf{v}_{h}\right) \in \mathbf{U}_{h} \times \mathbf{U}_{h}$,

$$
a_{h}\left(\mathbf{w}_{h}, \mathbf{v}_{h}\right)=\int_{\Omega} \underline{\underline{\Sigma_{h}^{0}}}\left(\mathbf{w}_{h}\right): \underline{\underline{E_{h}^{0}}}\left(\mathbf{v}_{h}\right)+j_{h}\left(\mathbf{w}_{h}, \mathbf{v}_{h}\right)
$$


with

$$
\begin{aligned}
j_{h}\left(\mathbf{w}_{h}, \mathbf{v}_{h}\right):= & -\int_{\Omega}\left(2 \mu \underline{\underline{R_{h}^{0}}}\left(\mathbf{w}_{h}\right): \underline{\underline{R_{h}^{0}}}\left(\mathbf{v}_{h}\right)+\lambda R_{h}^{0}\left(\mathbf{w}_{h}\right) R_{h}^{0}\left(\mathbf{v}_{h}\right)\right) \\
& +\sum_{F \in \mathcal{F}_{h}} \int_{\Omega} \eta\left(2 \mu \underline{\underline{r_{F}^{0}}}\left(\llbracket \mathbf{w}_{h} \rrbracket\right): \underline{\underline{r_{F}^{0}}}\left(\llbracket \mathbf{v}_{h} \rrbracket\right)+\lambda r_{F}^{0}\left(\llbracket \mathbf{w}_{h} \rrbracket\right) r_{F}^{0}\left(\llbracket \mathbf{v}_{h} \rrbracket\right)\right) \\
& +\sum_{F \in \mathcal{F}_{h}} \int_{F} \frac{\gamma_{\mu, F}}{h_{F}} \llbracket \mathbf{w}_{h} \rrbracket \cdot \llbracket \mathbf{v}_{h} \rrbracket .
\end{aligned}
$$

Remark 13 (Extension to $\mathbf{U}_{* h} \times \mathbf{U}_{* h}$ ). An important remark is that the expressions 12 ) and $(20)$ are equivalent at the discrete level only, i.e., the extension of (20) to $\mathbf{U}_{* h} \times \mathbf{U}_{* h}$ does not coincide with (12). Such an extension is not required in the proof of Theorem 15.

Remark 14 (Coercivity). Letting $\mathbf{w}_{h}=\mathbf{v}_{h}$ in 20 and assuming $\eta>N_{\partial}$, coercivity holds in the form

$$
\begin{aligned}
a_{h}\left(\mathbf{v}_{h}, \mathbf{v}_{h}\right) & \geq\left\|(2 \mu)^{1 / 2} \underline{\underline{E_{h}^{0}}}\left(\mathbf{v}_{h}\right)\right\|_{L^{2}(\Omega)^{d, d}}^{2}+\left\|\lambda^{1 / 2} D_{h}^{0}\left(\mathbf{v}_{h}\right)\right\|_{L^{2}(\Omega)}^{2}+\left(\eta-N_{\partial}\right)\left(\left|\mathbf{v}_{h}\right|_{\mathrm{r}, \mu}^{2}+\left|\mathbf{v}_{h}\right|_{\mathrm{r}, \lambda}^{2}\right)+\left|\mathbf{v}_{h}\right|_{\mathrm{J}, \mu}^{2} \\
& :=\mid] \mathbf{v}_{h}\left[\left.\right|_{\mu, \lambda} ^{2}+\left(\eta-N_{\partial}\right)\left(\left|\mathbf{v}_{h}\right|_{\mathrm{r}, \mu}^{2}+\left|\mathbf{v}_{h}\right|_{\mathrm{r}, \lambda}^{2}\right)+\left|\mathbf{v}_{h}\right|_{\mathrm{J}, \mu}^{2} .\right.
\end{aligned}
$$

Theorem 15 (Convergence to minimal regularity solutions). Let $\left(\mathcal{T}_{h}\right)_{h \in \mathcal{H}}$ be an admissible mesh sequence, and denote by $\left(\mathbf{u}_{h}\right)_{h \in \mathcal{H}}$ the sequence of discrete solutions to problem 13 . Assume $\eta>N_{\partial}=d+1$. Then, with $\mathbf{u} \in H_{0}^{1}(\Omega)^{d}$ unique solution to (3), there holds

$$
\begin{aligned}
\mathbf{u}_{h} & \rightarrow \mathbf{u} & & \text { strongly in } L^{2}(\Omega)^{d}, \\
\underline{\underline{\epsilon_{h}}}\left(\mathbf{u}_{h}\right) & \rightarrow \epsilon(\mathbf{u}) & & \text { strongly in } L^{2}(\Omega)^{d, d}, \\
\operatorname{div}_{h} \mathbf{u}_{h} & \rightarrow \operatorname{div} \mathbf{u} & & \text { strongly in } L^{2}(\Omega), \\
\left|\mathbf{u}_{h}\right|_{\mathrm{r}, \mu}+\left|\mathbf{u}_{h}\right|_{\mathrm{r}, \lambda}+\left|\mathbf{u}_{h}\right|_{\mathrm{J}, \mu} & \rightarrow 0 . & &
\end{aligned}
$$

Proof. (i) A priori estimate. Using (15) together with the Cauchy-Schwarz and discrete Poincaré inequalities it is inferred

$$
\alpha_{\eta} \underline{\mu^{2}} C_{\mathrm{K}}\left\|\mathbf{u}_{h}\right\|_{1, h}^{2} \leq a_{h}\left(\mathbf{u}_{h}, \mathbf{u}_{h}\right)=\int_{\Omega} \mathbf{f} \cdot \mathbf{u}_{h} \leq C_{\mathrm{P}}\|\mathbf{f}\|_{L^{2}(\Omega)^{d}}\|\mathbf{u}\|_{1, h}
$$

hence the a priori estimate $\left\|\mathbf{u}_{h}\right\|_{1, h} \leq \frac{C_{\mathrm{P}}}{\alpha_{\eta} \mu^{2} C_{\mathrm{K}}}\|\mathbf{f}\|_{L^{2}(\Omega)^{d}}$ holds. Since $\left\|\mathbf{u}_{h}\right\|_{1, h}$ is bounded uniformly in $h$, owing to [18, Theorem 5.7], there exists a subsequence (not renumbered to alleviate the notation) and an element $\mathbf{w} \in H_{0}^{1}(\Omega)^{d}$ such that $\mathbf{u}_{h} \rightarrow \mathbf{w}$ strongly in $L^{2}(\Omega)^{d}$ and $\underline{\underline{G_{h}^{0}}}\left(\mathbf{u}_{h}\right) \rightarrow \nabla \mathbf{w}$ weakly in $L^{2}(\Omega)^{d, d}$. Hence, $\underline{\underline{E_{h}^{0}}}\left(\mathbf{u}_{h}\right) \rightarrow \underline{\underline{\epsilon}}(\mathbf{w})$ weakly in $L^{2}(\Omega)^{d, d}$ and $D_{h}^{0}(\mathbf{w}) \rightarrow$ div w weakly in $L^{2}(\Omega)$. (ii) Convergence of $\mathbf{u}_{h}$. Let $\mathbf{v} \in C^{\infty}(\Omega)^{d}$ and, for $h \in \mathcal{H}$, denote by $\mathbf{v}_{h}$ the Lagrange interpolate of $\mathbf{v}$ onto $\mathcal{T}_{h}$. Then, using $\mathbf{v}_{h}$ as a test function in $(13)$, since $\underline{\underline{E_{h}^{0}}}\left(\mathbf{v}_{h}\right) \rightarrow \underline{\underline{\epsilon}}(\mathbf{v})$ strongly in $L^{2}(\Omega)^{d, d}, D_{h}^{0}\left(\mathbf{v}_{h}\right) \rightarrow$ div $\mathbf{v}$ strongly in $L^{2}(\Omega)$, and $j_{h}\left(\mathbf{w}_{h}, \mathbf{v}_{h}\right)=0$, it is inferred from 20.

$$
\int_{\Omega} \underline{\underline{\sigma}}(\mathbf{w}): \underline{\underline{\epsilon}}(\mathbf{v}) \leftarrow a_{h}\left(\mathbf{w}_{h}, \mathbf{v}_{h}\right)=\int_{\Omega} \mathbf{f} \cdot \mathbf{v}_{h} \rightarrow \int_{\Omega} \mathbf{f} \cdot \mathbf{v} .
$$

By density of $C^{\infty}(\Omega)^{d}$ we conclude that $\mathbf{w}$ coincides with $\mathbf{u}$ unique solution to $(13)$. This implies that the whole sequence $\left(\mathbf{u}_{h}\right)_{h \in \mathcal{H}}$ converges and proves 22a. (iii) Convergence of $\underline{\underline{\epsilon_{h}}}\left(\mathbf{u}_{h}\right), \operatorname{div}_{h} \mathbf{u}_{h}$, and of the jumps. Using (21) it is inferred

$$
\left.\limsup _{h \rightarrow 0} \mid\right] \mathbf{u}_{h}\left[\left.\right|_{\mu, \lambda} ^{2} \leq \lim _{h \rightarrow 0} a_{h}\left(\mathbf{u}_{h}, \mathbf{u}_{h}\right)=\lim _{h \rightarrow 0} \int_{\Omega} \mathbf{f} \cdot \mathbf{u}_{h}=\int_{\Omega} \mathbf{f} \cdot \mathbf{u}=a(\mathbf{u}, \mathbf{u}) .\right.
$$




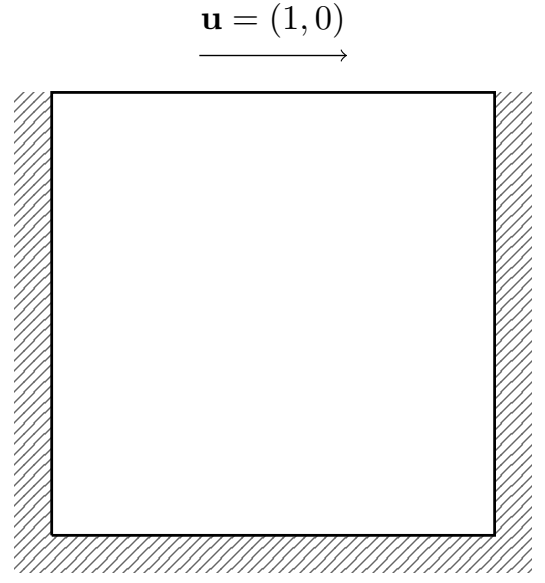

(a) Closed cavity

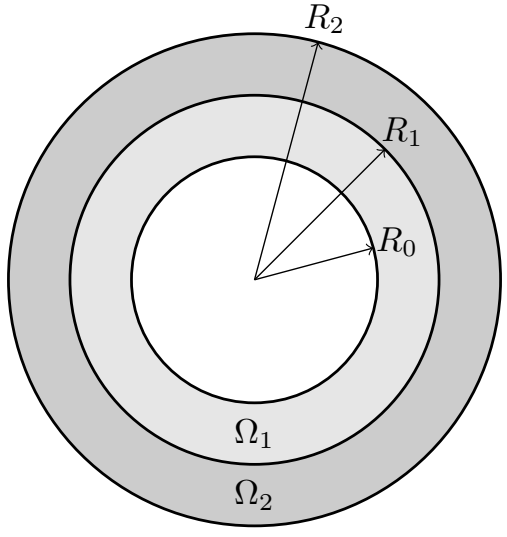

(b) Concentric cylindrical layers

Figure 1: Configurations for the numerical examples

On the other hand, owing to weak convergence,

$$
\left.\liminf _{h \rightarrow 0} \mid\right] \mathbf{u}_{h}\left[\left.\right|_{\mu, \lambda} \geq\left\|(2 \mu)^{1 / 2} \underline{\underline{\epsilon}}(\mathbf{u})\right\|_{L^{2}(\Omega)^{d, d}}^{2}+\left\|\lambda^{1 / 2} \operatorname{div} \mathbf{u}\right\|_{L^{2}(\Omega)}^{2}=a(\mathbf{u}, \mathbf{u}) .\right.
$$

Hence, we conclude $\left.\lim _{h \rightarrow 0} \mid\right] \mathbf{u}_{h}\left[\left.\right|_{\mu, \lambda}=a_{h}(\mathbf{u}, \mathbf{u})\right.$, which, together with the weak convergence of $\underline{\underline{E_{h}^{0}}}\left(\mathbf{u}_{h}\right)$ and $D_{h}^{0}\left(\mathbf{u}_{h}\right)$ proved in the previous point, classically yields $\underline{\underline{E_{h}^{0}}}\left(\mathbf{u}_{h}\right) \rightarrow \underline{\underline{\epsilon}}(\mathbf{u})$ strongly in $L^{2}(\Omega)^{d, d}$ and $D_{h}^{0}\left(\mathbf{u}_{h}\right) \rightarrow \operatorname{div} \mathbf{u}$ strongly in $L^{2}(\Omega)$. Using again (21) it is inferred

$$
\left.\left(\eta-N_{\partial}\right)\left(\left|\mathbf{u}_{h}\right|_{\mathrm{r}, \mu}+\left|\mathbf{u}_{h}\right|_{\mathrm{r}, \lambda}\right)+\left|\mathbf{u}_{h}\right|_{\mathrm{J}, \mu} \leq a_{h}\left(\mathbf{u}_{h}, \mathbf{u}_{h}\right)-\mid\right] \mathbf{u}_{h}\left[\left.\right|_{\mu, \lambda} ^{2},\right.
$$

and $(22 \mathrm{~d})$ follows. To prove $22 \mathrm{~b})$ and $(22 \mathrm{c})$ observe that, owing to the triangular inequality,

$$
\begin{gathered}
\left\|\underline{\underline{E_{h}^{0}}}\left(\mathbf{u}_{h}\right)\right\|_{L^{2}(\Omega)^{d, d}}-\left\|\underline{\underline{R_{h}^{0}}}\left(\mathbf{u}_{h}\right)\right\|_{L^{2}(\Omega)^{d, d}} \leq\left\|\underline{\underline{\epsilon_{h}}}\left(\mathbf{u}_{h}\right)\right\|_{L^{2}(\Omega)^{d, d}} \leq\left\|\underline{\underline{E_{h}^{0}}}\left(\mathbf{u}_{h}\right)\right\|_{L^{2}(\Omega)^{d, d}}+\left\|\underline{\underline{R_{h}^{0}}}\left(\mathbf{u}_{h}\right)\right\|_{L^{2}(\Omega)^{d, d}}, \\
\left\|D_{h}^{0}\left(\mathbf{u}_{h}\right)\right\|_{L^{2}(\Omega)}-\left\|R_{h}^{0}\left(\mathbf{u}_{h}\right)\right\|_{L^{2}(\Omega)} \leq\left\|\operatorname{div}_{h} \mathbf{u}_{h}\right\|_{L^{2}(\Omega)} \leq\left\|D_{h}^{0}\left(\mathbf{u}_{h}\right)\right\|_{L^{2}(\Omega)}+\left\|R_{h}^{0}\left(\mathbf{u}_{h}\right)\right\|_{L^{2}(\Omega)} .
\end{gathered}
$$

Since $\left\|\underline{\underline{R_{h}^{0}}}\left(\mathbf{u}_{h}\right)\right\|_{L^{2}(\Omega)^{d}} \leq(2 \underline{\mu})^{-1 / 2} N_{\partial}\left|\mathbf{u}_{h}\right|_{\mathrm{r}, \mu}$ and $\left\|R_{h}^{0}\left(\mathbf{u}_{h}\right)\right\|_{L^{2}(\Omega)} \leq \underline{\lambda}^{-1 / 2}\left|\mathbf{u}_{h}\right|_{\mathrm{r}, \lambda}$, the conclusion follows letting $h \rightarrow 0$ and using $(22 \mathrm{~d})$.

Remark 16 (General meshes). The above proof extends to general polyhedral meshes by replacing the Lagrange interpolator by the $L^{2}$-orthogonal projector. For further details see [18, Sect. 5.2] and [18, Chapter 1].

\section{Numerical examples}

\subsection{Closed cavity}

Our first test case is the homogeneous closed cavity problem proposed by Hansbo and Larson [3. The domain is the unit square $\Omega=(0,1)^{2}$. A horizontal displacement is imposed on the upper side, whereas the others are kept fixed; see Figure 1(a). The elastic modulus and Poisson's ratio are chosen as $E=1000$ and $\nu=0.4999$. The values of the Lamé parameters are obtained from the relations

$$
\lambda=\frac{\nu E}{(1+\nu)(1-2 \nu)}, \quad \mu=\frac{E}{2(1+\nu)} .
$$

Two uniform triangular meshes with meshsize $h \in\left\{6.25 \cdot 10^{-2}, 1.5625 \cdot 10^{-2}\right\}$ are used, and a comparison with the stabilized Crouzeix-Raviart method of [3] is provided for the sake of completeness. The values of the horizontal displacement along the vertical centerline and of the vertical 


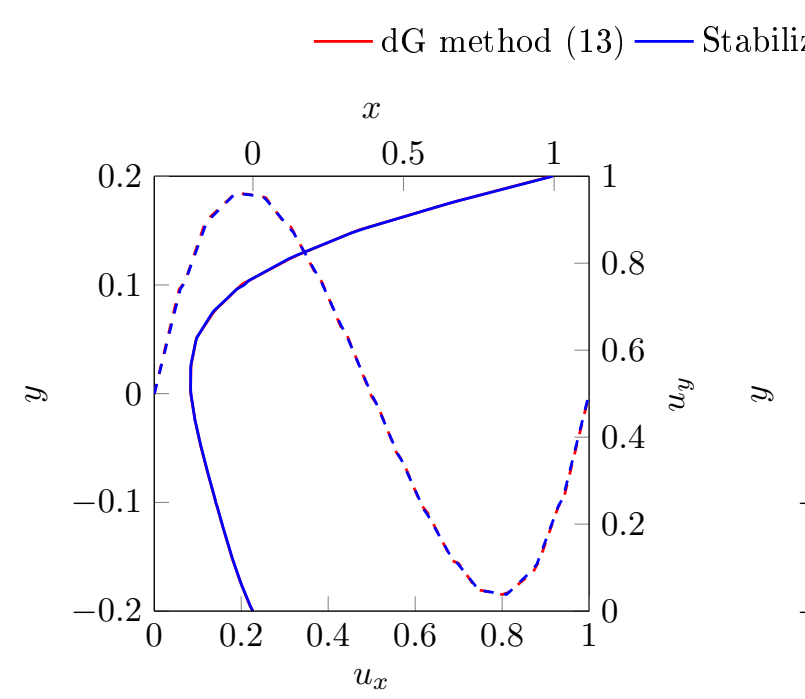

(a) Coarse mesh

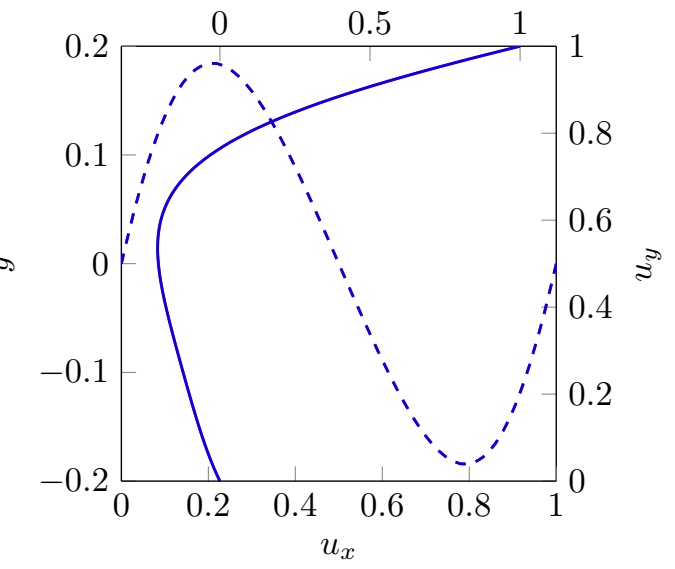

(b) Fine mesh

Figure 2: Results for the closed cavity problem. Solid lines: $x$-component of the displacement along the vertical centerline. Dashed lines: $y$-component of the displacement along the horizontal centerline.

displacement along the horizontal centerline are depicted in Figure 2, The methods show good agreement, and no sign of locking is present in both cases.

\subsection{Concentric cylindrical layers}

To test the robustness with respect to the heterogeneity of the medium we consider the exact solution derived in 9 for a configuration composed of concentric hollow cylindrical layers subject to a homogeneous pressure $q$ on the inner surface and $Q$ on the outer surface. The configuration is depicted in Figure 1(b). Denote by $\mathfrak{B}_{R}$ the ball of radius $R>0$ centered in the origin. For three

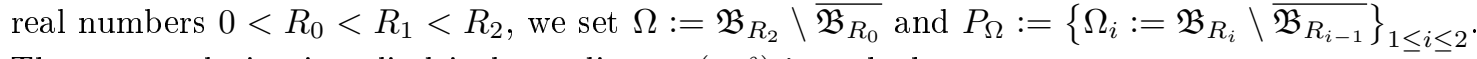
The exact solution in cylindrical coordinates $(r, \vartheta)$ is such that

$$
\mathbf{u}_{\mid \Omega_{i}}=\left(\frac{\alpha_{i} A_{i}}{r}+\beta_{i} B_{i} r\right) \mathbf{r}, \quad 1 \leq i \leq 2
$$

where $\mathbf{r}$ denotes the unit vector in the radial direction and, for a given pressure module $q$, the coefficients $\left\{\alpha_{i}, \beta_{i}\right\}_{1 \leq i \leq 2}$ are such that

$$
\alpha_{i}=-\frac{1+\nu_{i}}{E_{i}}, \quad \beta_{i}=\frac{\left(1-2 \nu_{i}\right)\left(1+\nu_{i}\right)}{E_{i}}, \quad 1 \leq i \leq 2 .
$$

Here, $E_{i}$ and $\nu_{i}$ denote, respectively, the constant values of the elastic modulus and Poisson's ratio, while the coefficients $\left\{A_{i}, B_{i}\right\}_{1 \leq i \leq 2}$ solve

$$
\begin{aligned}
& \frac{A_{1}}{R_{0}^{2}}+B_{1}=-q, \quad \frac{\alpha_{2}-\alpha_{1}}{\alpha_{2}-\beta_{2}} \frac{A_{1}}{R_{1}^{2}}+\frac{\alpha_{2}-\beta_{1}}{\alpha_{2}-\beta_{2}} B_{1}-B_{2}=0, \\
& \frac{A_{2}}{R_{2}^{2}}+B_{2}=-Q, \quad \frac{\alpha_{1}-\beta_{2}}{\alpha_{2}-\beta_{2}} A_{1}-A_{2}+\frac{\beta_{1}-\beta_{2}}{\alpha_{2}-\beta_{2}} B_{1} R_{1}^{2}=0 .
\end{aligned}
$$

The constant values of the Lamé parameters $\lambda_{i}$ and $\mu_{i}$ in each layer $\Omega_{i}, i \in\{1,2\}$ can be inferred from (23). For the tests we have used the following values: $R_{0}=0.5, R_{1}=0.75, R_{2}=1, E_{i}=1$ for $1 \leq i \leq 2, \nu_{1}=1, \nu_{2} \in\{0.499,0.4999,0.49999\}$. The stabilization parameter $\eta$ is taken equal to 1 . To study the convergence, we use a sequence of uniformly refined unstructured triangular meshes $\left(\mathcal{T}_{h_{j}}\right)_{0 \leq j \leq 4}$ with $h_{j}:=h_{0} / 2^{j}$ and $h_{0}=0.25$. The results displayed in Figure 3 show that the precision is not affected when the outer layer tends to the incompressible limit. 


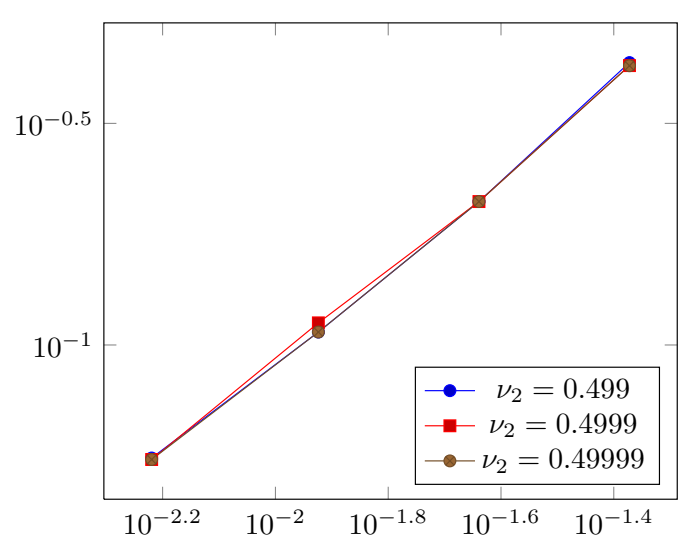

(a) $\left\|\mathbf{u}-\mathbf{u}_{h}\right\|_{\mu, \lambda}$ vs. $h$

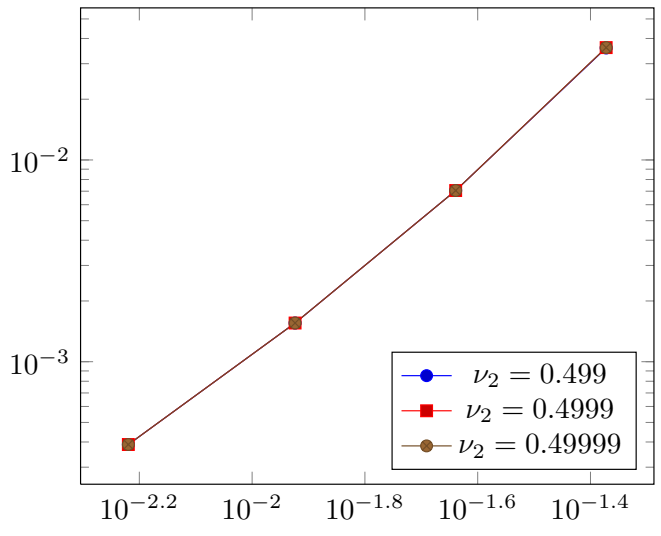

(b) $\left\|\mathbf{u}-\mathbf{u}_{h}\right\|_{L^{2}(\Omega)^{d}}$ vs. $h$

Figure 3: Errors versus mesh sizes

Remark 17 (Smooth domains). The theory of Sect. 2 can be extended to the smooth domain of Figure 1(b), In practice, however, smooth domains are often approximated by polygonal domains at the numerical levels, so that the case considered in Sect. 2 turns out to be the most important in practice.

[1] S. Brenner, L.-Y. Sung, Linear finite element methods for planar linear elasticity, Math. Comp. 49 (200) (1992) 321-338.

[2] P. Hansbo, M. G. Larson, Discontinuous Galerkin methods for incompressible and nearly incompressible elasticity by Nitsche's method, Comput. Methods Appl. Mech. Engrg. 191 (2002) 1895-1908.

[3] P. Hansbo, M. G. Larson, Discontinuous Galerkin and the Crouzeix-Raviart element: Application to elasticity, M2AN Math. Model. Numer. Anal. 37 (1) (2003) 63-72.

[4] T. P. Wihler, Locking-free adaptive discontinuous Galerkin FEM for linear elasticity problems, Math. Comp. 75 (255) (2006) 1087-1102.

[5] S. Nicaise, D. Mercier, Regularity results of Stokes/Lamé interface problems, Math. Nachrichten 285 (2012) 332-348.

[6] S. C. Brenner, Korn's inequalities for piecewise $H^{1}$ vector fields, Math. Comp. 73 (247) (2004) 1067-1087.

[7] F. Bassi, S. Rebay, A high-order accurate discontinuous finite element method for the numerical solution of the compressible Navier-Stokes equations, J. Comput. Phys. 131 (2) (1997) $267-279$.

[8] F. Bassi, S. Rebay, G. Mariotti, S. Pedinotti, M. Savini, A high-order accurate discontinuous finite element method for inviscid and viscous turbomachinery flows, in: R. Decuypere, G. Dibelius (Eds.), Proceedings of the $2^{\text {nd }}$ European Conference on Turbomachinery Fluid Dynamics and Thermodynamics, 1997, pp. 99-109.

[9] X. Hongjun, S. Zhifei, Z. Taotao, Elastic analyses of heterogeneous hollow cylinders, Mechanics Research Communications 33 (2006) 681-691.

[10] P. Grisvard, Elliptic problems in nonsmooth domains, Vol. 24 of Monographs and Studies in Mathematics, Pitman, Boston-London-Melbourne, 1985. 
[11] M. Dauge, Elliptic boundary value problems on corner domains - smoothness and asymptotics of solutions, Vol. 1341 of Lecture Notes in Mathematics, Springer, Berlin, 1988.

[12] M. Orlt, A.-M. Sändig, Regularity of viscous Navier-Stokes flows in nonsmooth domains, in: M. Costabel, M. Dauge, S. Nicaise (Eds.), Boundary value problems and integral equations in nonsmooth domains, Vol. 167 of Lecture Notes in Pure and Applied Mathematics, Marcel Dekker, New York, 1995, pp. 101-120.

[13] D. Mercier, Problèmes de transmission sur des réseaux polygonaux pour des systèmes d'edp, Annales de la faculté des Sciences de Toulouse X (1) (2001) 107-162.

[14] S. Nicaise, A.-M. Sändig, General interface problems. I, II Math. Methods Appl. Sci. 17 (6) (1994) 395-429, 431-450. doi:10.1002/mma.1670170602

URL http://dx.doi.org/10.1002/mma.1670170602

[15] P. G. Ciarlet, The finite element method for elliptic problems, Vol. 40 of Classics in Applied Mathematics, Society for Industrial and Applied Mathematics (SIAM), Philadelphia, PA, 2002, reprint of the 1978 original [North-Holland, Amsterdam; MR0520174 (58 \#25001)].

[16] F. Brezzi, G. Manzini, L. D. Marini, P. Pietra, A. Russo, Discontinuous Galerkin approximations for elliptic problems, Numer. Methods Partial Differential Equations 16 (4) (2000) 365-378.

[17] D. A. Di Pietro, A. Ern, Analysis of a discontinuous Galerkin method for heterogeneous diffusion problems with low-regularity solutions, Numer. Meth. PDEs.Published online. DOI: $10.1002 /$ num.20675.

[18] D. A. Di Pietro, A. Ern, Mathematical aspects of discontinuous Galerkin methods, Vol. 69 of Mathématiques \& Applications, Springer-Verlag, Berlin, 2011.

[19] P. Monk, E. Süli, The adaptive computation of far-field patterns by a posteriori error estimation of linear functionals, SIAM J. Numer. Anal. 36 (1) (1999) 251-274.

[20] C. Carstensen, S. A. Funken, Constants in Clément-interpolation error and residual based a posteriori error estimates in finite element methods, East-West J. Numer. Math. 8 (3) (2000) $153-175$.

[21] M. Vohralík, On the discrete Poincaré-Friedrichs inequalities for nonconforming approximations of the Sobolev space $H^{1}$, Numer. Funct. Anal. Optim. 26 (7-8) (2005) 925-952.

[22] D. A. Di Pietro, A. Ern, Discrete functional analysis tools for discontinuous Galerkin methods with application to the incompressible Navier-Stokes equations, Math. Comp. 79 (2010) 13031330 . 